\title{
Correction to: Modularity lifting beyond the Taylor-Wiles method
}

\author{
Frank Calegari ${ }^{1}$. David Geraghty ${ }^{2}$
}

Published online: 31 December 2021

(C) Springer-Verlag GmbH Germany, part of Springer Nature 2021

\section{Correction to: Invent. math. 211:297-433, https://doi.org/10.1007/s00222-017-0749-x}

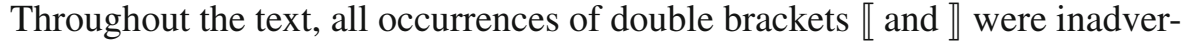
tently replaced by single brackets. This occurred 47 times, resulting in 47 errors to the final manuscript. The error was introduced by a typesetting operator. To highlight some specific points where confusion could arise:

(1) The ring $S_{\infty}$ of $\S 2$ (and thereafter) should be isomorphic to the completed group ring $\mathcal{O} \llbracket\left(\mathbf{Z}_{p}\right)^{q} \rrbracket$ rather than the polynomial ring $\mathcal{O}\left[\left(\mathbf{Z}_{p}\right)^{q}\right]$. This also occurs in the proof of Proposition 2.3, the introduction to $\S 6$, in Theorem 6.3, and also in Theorem 9.13. (5 occurrences)

(2) The ring $R_{\infty}$ of Proposition 2.3 should be defined as the power series ring $\mathcal{O} \llbracket x_{1}, \ldots, x_{q-1} \rrbracket$ (or possibly a different number of variables when it occurs later in the text) rather than the polynomial ring $\mathcal{O}\left[x_{1}, \ldots, x_{q-1}\right]$ (5 occurrences)

The online version of the original article can be found under https://doi.org/10.1007/s00222017-0749-x.

$凶$ Frank Calegari

fcale@math.uchicago.edu

David Geraghty

djgeraghty@gmail.com

1 The University of Chicago, 5734 S University Ave, Chicago, IL 60637, USA

2 Department of Mathematics, Carney Hall, Boston College, Chestnut Hill, MA 02467-

3806, USA 
(3) The $q$-expansion map appearing in the proof of Theorem 3.11 should be valued in the module $K / \mathcal{O} \llbracket q \rrbracket$ rather than $K / \mathcal{O}[q]$. (2 occurrences)

(4) The explicit presentation of a local deformation ring over $\mathcal{O} / \varpi^{m}$ in the proof of Lemma 3.22 should be a quotient of a power series ring. (1 occurrence)

(5) The completed local rings of the modular curves considered in the proof of Lemma 3.28 should be $\mathcal{O} \llbracket q \rrbracket$ (or $\mathcal{O} \llbracket q^{e} \rrbracket$ ) rather than the rings $\mathcal{O}[q]$ or $\mathcal{O}\left[q^{e}\right]$. (9 occurrences)

(6) The power series ring $A$ in the statement of Lemma 4.2 has the form $B \llbracket T_{1}, \ldots T_{n} \rrbracket$ and not $B\left[T_{1}, \ldots, T_{n}\right]$. Similarly, all other rings occurring in the proof of this Lemma should be power series rings and not polynomial rings. (5 occurrences)

(7) Various presentations of rings related to $\widetilde{R}^{\dagger}$ in the proof of Theorem 4.3 should be power series rings over $k$ rather than polynomial rings. ( 5 occurrences)

(8) The generating function $H_{R}(t)$ in the proof of Theorem 4.3 should be defined as an element of $\mathbf{Z} \llbracket t \rrbracket$ and not of the ring $\mathbf{Z}[t]$. (2 occurrences)

(9) The framed deformation rings occurring before Lemma 4.11 should be quotients of power series rings over local deformation rings, as should some of the explicit rings occurring in the proof of Lemma 4.11. $(6=$ $3+3$ occurrences)

(10) The ring $\mathcal{O}^{\square}$ in Theorem 6.3 should be a power series ring, not a polynomial ring. (1 occurrence)

(11) The ring $A[X]$ occurring in the statement of Cor 9.13 should be $A \llbracket X \rrbracket$. However, the ring $A[T] /\left(T^{q}-1\right)$ is indeed a quotient of a polynomial ring rather than a power series ring. This may be the only point in the paper where it would not be correct to replace any occurrence of a polynomial ring with a power series ring. (1 occurrence)

(12) The rings $\mathcal{O}^{\square}, R_{\infty}^{1}$ and $R_{\infty}^{2}$ in Theorem 9.19 should be power series rings. (5 occurrences)

Publisher's Note Springer Nature remains neutral with regard to jurisdictional claims in published maps and institutional affiliations. 